\title{
Exosomal microRNA-301a-3p promotes the proliferation and invasion of nasopharyngeal carcinoma cells by targeting BTG1 mRNA
}

\author{
QIANG CHENG, QIANG LI, LINGEN XU and HUI JIANG \\ Department of Otolaryngology, Jinshan Hospital Affiliated to Fudan University, Shanghai 201508, P.R. China
}

Received September 19, 2020; Accepted February 12, 2021

DOI: $10.3892 / \mathrm{mmr} .2021 .11967$

\begin{abstract}
Aberrant microRNA (miRNA/miR) expression plays an important role in the pathogenesis of nasopharyngeal carcinoma (NPC). In the present study, the role and underlying molecular mechanism of miR-301a-3p in NPC cells were determined. It was observed that miR-301a-3p upregulation promoted NPC cell proliferation, migration, invasion and epithelial-mesenchymal transition in vitro, whereas its downregulation resulted in the opposite effect. B-cell translocation gene 1 (BTG1) mRNA was identified as the novel target of miR-301a-3p. BTG1 overexpression partially attenuated miR-301a-3p-induced increase in cell proliferation and invasion. miR-301a-3p can be transferred by exosomes and positively regulate the proliferation and invasion of NPC cells. Altogether, the present study highlights that exosomal miR-301a-3p can promote NPC cell proliferation and invasion by repressing BTG1, thereby resulting in the development of NPC.
\end{abstract}

\section{Introduction}

Nasopharyngeal carcinoma (NPC), a highly malignant tumor originating from the nasopharyngeal epithelium, is among the most prevalent malignancies in China (1). The combined use of magnetic resonance imaging and intensity-modulated radiotherapy, as well as advanced radiotherapy and chemotherapy, have substantially improved the prognosis of patients with NPC. However, numerous patients are diagnosed with advanced NPC, and many develop distant metastases and recurrent disease within 4 years of treatment (2). To date, the pathogenesis of NPC has not been fully elucidated. Therefore, further research to determine the pathogenesis of NPC and to

Correspondence to: Dr Hui Jiang, Department of Otolaryngology, Jinshan Hospital Affiliated to Fudan University, 1508 Longhang Road, Shanghai 201508, P.R. China

E-mail: ns_j50665@sina.com

Key words: nasopharyngeal carcinoma, microRNA-301a-3p, B-cell translocation gene 1, proliferation, invasion elucidate a potential molecular marker for early diagnosis is urgently warranted.

MicroRNAs (miRNAs/miRs) act as tumor suppressor genes or oncogenes to modulate tumor progression, and may provide a new approach to explore the molecular mechanisms of the pathogenesis of NPC (3). miRNAs are a diverse class of short ( 22 nucleotides) non-coding RNAs that can regulate gene expression by attenuating translation or promoting mRNA degradation in a sequence-specific manner. miRNA dysfunction partially contributes to the development of cancer (4,5). Aberrant miRNA expression has been observed in patients with NPC. For example, miR-125b has been reported to be significantly upregulated in patients with NPC and has been identified as an independent predictor of poor patient survival. Furthermore, miR-125b functionally promoted proliferation and inhibited the apoptosis of NPC cells by targeting the $\mathrm{A} 20 / \mathrm{NF}-\kappa \mathrm{B}$ signaling pathway (6). Exosomal miR-24-3p impedes T-cell function by targeting fibroblast growth factor 11 and serves as a potential prognostic biomarker of NPC (7). Metastasis-associated miR-23a from NPC-derived exosomes promotes angiogenesis by targeting TSGA10 (8). Notably, miR-301a-3p is upregulated and functions as a potential oncogenic RNA in some types of cancer, including lung cancer, pancreatic cancer, prostate cancer and glioma (9-12). Nonetheless, the role of miR-301a-3p and its underlying mechanism in the progression of NPC remain poorly understood.

The present study demonstrated that miR-301a-3p upregulation promotes NPC cell proliferation, migration, invasion and epithelial-mesenchymal transition (EMT) in vitro, whereas downregulation has an opposite effect. In addition, it was identified that B-cell translocation gene 1 (BTG1) was the direct functional target of miR-301a-3p in NPC cells. Finally, it was shown that miR-301a-3p participates in cell-cell communication via exosomes. These findings reveal the novel mechanism of NPC progression and provide a molecular target for NPC research.

\section{Materials and methods}

Cell culture. Two NPC cell lines, C666-1 [Epstein-Barr virus (EBV+)] and 5-8F (EBV-), and an immortalized nasopharyngeal epithelial cell line (NP69) were procured from the Central South University (Hunan, China). C666-1 and 
5-8F cells were maintained in RPMI-1640 medium and supplemented with $10 \%$ fetal bovine serum (both from Gibco; Thermo Fisher Scientific, Inc.). NP-69 cells were cultured in keratinocyte serum-free medium supplemented with human recombinant epidermal growth factor and bovine pituitary extract (Sigma-Aldrich; Merck KGaA). All cells were cultured in a humidified chamber with $5 \% \mathrm{CO}_{2}$ at $37^{\circ} \mathrm{C}$.

Reverse transcription-quantitative polymerase chain reaction $(R T-q P C R)$. Total RNA was extracted from the cells and subjected to reverse transcription for $60 \mathrm{~min}$ at $37^{\circ} \mathrm{C}$ using the NCode miRNA First-Strand cDNA Synthesis kit (Thermo Fisher Scientific, Inc.). RT-qPCR was performed using the ABI 7900HT system with SYBR-Green PCR Master mix (Applied Biosystems; Thermo Fisher Scientific, Inc.) using the following thermocycling parameters: $95^{\circ} \mathrm{C}$ for $10 \mathrm{~min}$, followed by 40 cycles of $95^{\circ} \mathrm{C}$ for $10 \mathrm{sec}, 60^{\circ} \mathrm{C}$ for $20 \mathrm{sec}$ and $72^{\circ} \mathrm{C}$ for $35 \mathrm{sec}$. GAPDH and $U 6$ snRNA were used as internal controls. The data from each sample were normalized with the internal control, and fold changes were calculated via relative quantification $\left(2^{-\Delta \Delta \mathrm{Cq}}\right)(13)$. The primer sequences used were as follows: $m i R-301 a-3 p$ forward, 5'-CAG TGCAATAGTATTGT-3' and reverse, 5'-GTGCAGGGTCCGA GGT-3'; U6 forward, 5'-GCGCGTCGTGAAGCGTTC-3' and reverse, 5'-GTGCAGGGTCCGAGGT-3'; BTG1 forward, 5'-ACC GTTGTATTCGCATCA-3' and reverse,5'-CCATCCTCTCCAAT TCTGTA-3'; and GAPDH forward, 5'-ACAACTTTGGTATCGT GGAAGG-3' and reverse, 5'-GCCATCACGCCACAGTTTC-3'.

Transfection experiments. miR-301a-3p mimic (5'-CAGUGC AAUAGUAUUGUCAAAGC-3') and negative control (NC mimic, 5'-UCUACUCUUUCUAGGAGGUUGUGA-3') were purchased from Guangzhou RiboBio Co., Ltd. miR-301a-3p inhibitor (5'-GCUUUGACAATCTATTGCACTG-3') and negative control (NC-in, 5'-ACCGCUAAUCAUACGAAUA CAC-3') were purchased from Guangzhou RiboBio Co., Ltd. Plasmids (pcDNA3.1) expressing BTG1 and lentiviruses expressing miR-301a-3p were purchased from Hanbio Biotechnology Co., Ltd. Oligonucleotide (100 nM) transfection was performed using Lipofectamine ${ }^{\circledR} 2000$ (Thermo Fisher Scientific, Inc.). After 48 h of transfection, cells were collected for further investigation.

Cell proliferation, EdU and colony formation assays. To determine the effect of miR-301a-3p on cell proliferation, Cell Counting Kit-8 (CCK-8; Dojindo Molecular Technologies, Inc.) was used. Transfected cells were plated at $2 \times 10^{3}$ cells per well in 96-well plates in triplicate. At various time intervals, the number of cells per well was calculated as absorbance units at a wavelength of $450 \mathrm{~nm}$.

The EdU assay was performed using the EdU-594 Cell Proliferation kit (Beyotime Institute of Biotechnology). Transfected cells were plated at $3 \times 10^{5}$ cells per well in 6 -well plates. After $12 \mathrm{~h}$, cells were then washed with PBS and fresh medium containing $10 \mu \mathrm{M}$ EdU was added. Cells were subsequently incubated for $2 \mathrm{~h}$ at $37^{\circ} \mathrm{C}$ in $5 \% \mathrm{CO}_{2}$ and washed with PBS to remove the free EdU probe and medium. Cells were then fixed in $4 \%$ paraformaldehyde at room temperature for $15 \mathrm{~min}$ and stained with DAPI at room temperature for $5 \mathrm{~min}$. Images were captured using a fluorescence microscope (magnification, x200).
For the colony formation assay, 300 cells were seeded into individual wells of a 6 -well plate and cultured at $37^{\circ} \mathrm{C}$ for 12 days. The colony counts per well were determined after staining the cells with $0.1 \%$ crystal violet at room temperature for $30 \mathrm{~min}$. Images were captured using a Nikon camera (Nikon Corporation). Only unambiguous colonies (diameter $>40 \mu \mathrm{m})$ in the wells were analyzed using ImageJ software (version 1.49p; National Institutes of Health).

Transwell assay. Transwell assays were performed as previously described (10). Transfected cells $\left(1 \times 10^{5}\right)$ in serum-free RPMI-1640 medium were placed into either control inserts or Transwell chambers with or without Matrigel. A medium with $15 \%$ fetal bovine serum was placed in the lower chamber as a chemoattractant. After incubation at $37^{\circ} \mathrm{C}$ for $12 \mathrm{~h}$, cells that adhered to the lower surfaces of the membrane inserts were fixed in $4 \%$ paraformaldehyde at room temperature for $30 \mathrm{~min}$, stained with $0.1 \%$ crystal violet at room temperature for $2 \mathrm{~h}$, and counted under a microscope (magnification, $\mathrm{x} 200$; Olympus Corporation).

Western blotting. Western blotting was performed as previously described (8). In brief, protein lysates (10-30 $\mu \mathrm{g}$ ) were separated via sodium dodecyl sulfate-polyacrylamide gel electrophoresis and were subsequently transferred onto polyvinylidene difluoride membranes (EMD Millipore). The membranes were first incubated overnight with primary antibodies specific for E-cadherin (1:10,000; cat. no. 20874-1-AP), vimentin (1:5,000; cat. no. 10366-1-AP), BTG1 (1:1,000; cat. no. 14879-1-AP), CD63 (1:1,000; cat. no. 25682-1-AP), TSG101 (1:3,000; cat. no. 14497-1-AP) and GAPDH (1:6,000; cat. no. 60004-1-Ig) that had been diluted in a 5\% low-fat milk-TBS with $0.1 \%$ Tween-20 solution and then with a horseradish peroxidase-conjugated secondary antibody $(1: 3,000$; cat. no. PR30009). The labeled protein bands were visualized using enhanced chemiluminescence (Beyotime Institute of Biotechnology), and band intensities were semi-quantified using ImageJ software (version 1.49p; National Institutes of Health). All primary and secondary antibodies were purchased from Wuhan Sanying Biotechnology.

Target prediction. Targets of miR-301a-3p were searched for using different computational methods, such as miRanda (http://www.microrna.org/) and TargetScan (http://www. targetscan.org/vert_71/). These methods identified numerous candidate genes that were commonly predicted to be possible targets of miR-301a-3p. Gene Ontology (http://www.geneontology.org/) analysis was then carried out. Genes classified as having tumor-suppressive functions involved in NPC were identified; BTG1 was of particular interest due to its negative roles in cancer cell proliferation and invasion.

Luciferase reporter assay. The pMIR-REPORT vector (Promega Corporation) was used to generate luciferase reporters carrying either the wild-type (Wt) or mutated (Mut) 3'-untranslated region (UTR) of BTG1. C666-1 and 5-8F cells $\left(2 \times 10^{4}\right)$ were first seeded in 24-well plates, incubated for $24 \mathrm{~h}$, and then transfected with the miR-301a-3p mimic (100 $\mathrm{nM})$ and reporter vectors $(30 \mathrm{nM})$ at $37^{\circ} \mathrm{C}$ using Lipofectamine ${ }^{\circledR} 2000$. After $48 \mathrm{~h}$ of incubation, luciferase activity was analyzed 

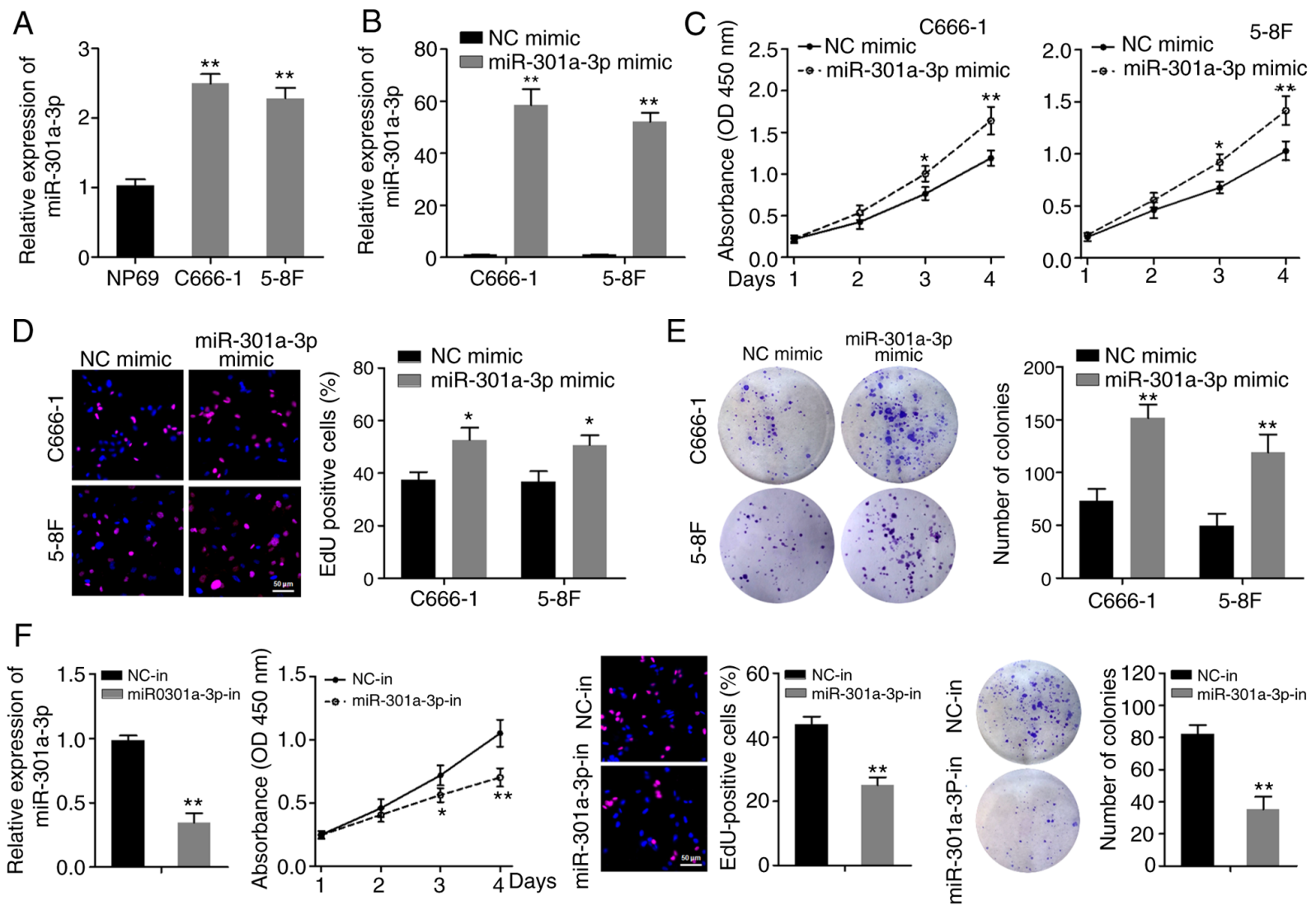

Figure 1. miR-301a-3p promotes NPC proliferation in vitro. (A) The expression of miR-301a-3p in two NPC cell lines (C666-1 and 5-8F) and the NP69 cell line was examined via RT-qPCR. (B) RT-qPCR analysis of miR-301a-3p in C666-1 and 5-8F cells after transfection of miR-301a-3p mimic or NC. (C) The proliferation of C666-1 and 5-8F cells after transfection with the miR-301a-3p mimic or NC was assessed using the Cell Counting Kit-8 assay. (D) EdU assay of C666-1 and 5-8F cells transfected with miR-301a-3p mimic or NC. Magnification, x200. (E) Representative images (left) and quantification (right) of the indicated cells subjected to the colony-formation assay. (F) miR-301a-3p inhibition prevents C666-1 cell proliferation and decreases EdU-positive rate and clonogenic potential. Magnification, $\mathrm{x} 200 .{ }^{*} \mathrm{P}<0.05 ;{ }^{* *} \mathrm{P}<0.01$ vs. Control group. NPC, nasopharyngeal carcinoma cell; miR, microRNA; RT-qPCR, reverse transcription-quantitative PCR; $\mathrm{NC}$, negative control; in, inhibitor.

using the Dual-Luciferase Reporter Assay System (Promega Corporation).

Exosome separation. Exosomes were separated from the cell culture medium using a previously described method (14). Briefly, the culture supernatants from C666-1 cells that stably overexpress miR-301a-3p were differentially centrifuged at $300 \mathrm{x} \mathrm{g}, 1,200 \mathrm{x} \mathrm{g}$ and $10,000 \mathrm{x} \mathrm{g}$ for $3 \mathrm{~h}$ at $4^{\circ} \mathrm{C}$. The supernatant obtained was then filtered and ultracentrifuged at $110,000 \mathrm{x} \mathrm{g}$ for $3 \mathrm{~h}$ at $4^{\circ} \mathrm{C}$. Exosomes were collected and resuspended in PBS.

Transmission electron microscopy (TEM) observation. Isolated exosomes were diluted with PBS, and $10 \mu \mathrm{l}$ sample was absorbed to a copper mesh. Then, the solution on the surface of the copper mesh was absorbed with filter paper after being placed for $5 \mathrm{~min}$. The copper mesh was cleaned once and dyed for $45 \mathrm{sec}$ with $2 \% 10 \mu \mathrm{l}$ uranyl acetate. The image was developed with $80-120 \mathrm{kV}$ projection electron microscope (JEM-1230; JEOL). Digital images were collected with a Gatan model 830 ORIUS SC200 CCD camera using Gatan Digital Micrograph software version 3.11 (Gatan, Inc.).

Statistical analyses. The SPSS 17.0 software (SPSS, Inc.) was used for statistical analyses. All data are presented as the means \pm standard deviations from at least three independent experiments. The differences between groups were analyzed using unpaired Student's t-test. Comparisons among multiple groups were analyzed by one-way analysis of variance (ANOVA), followed by Tukey's post hoc test. $\mathrm{P}<0.05$ was considered to indicate a statistically significant difference.

\section{Results}

miR-301a-3p is upregulated in NPC cell lines and promotes NPC cell proliferation in vitro. RT-qPCR was first used to examine the expression levels of miR-301a-3p in two NPC cell lines (C666-1 and 5-8F) and in the NP69 immortalized normal human nasopharynx epithelial cell line. As shown in Fig. 1A, miR-301a-3p was expressed at higher levels in NPC cell lines compared with in NP69 cells. Subsequently, the function of miR-301a-3p in vitro was explored via gain- and loss-of-function assays, using miR-301a-3p mimics and a specific inhibitor. The CCK-8 assay revealed that overexpression of miR-301a-3p enhanced the proliferative abilities of C666-1 and 5-8F cells (Fig. 1B and C). The EdU assay revealed that the EdU-positive rate was significantly increased in NPC cells when miR-301a-3p was overexpressed (Fig. 1D). Furthermore, miR-301a-3p overexpression increased the colony-formation efficiency of C666-1 and 5-8F cells (Fig. 1E). Accordingly, C666-1 cells subjected to miR-301a-3p knockdown exhibited decreased cell proliferation 

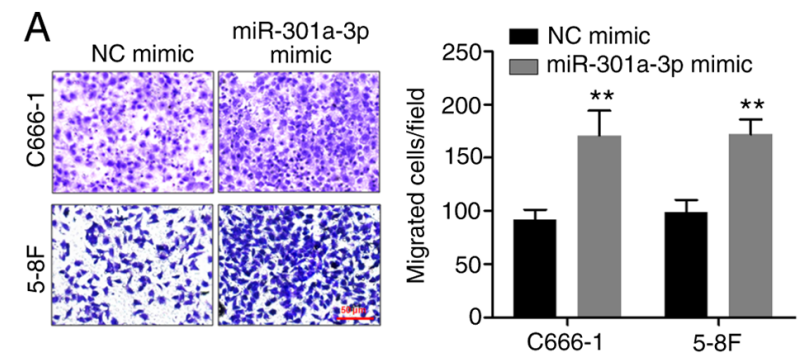

C
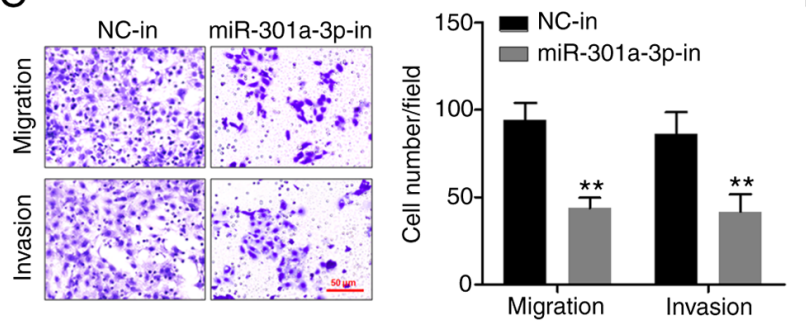
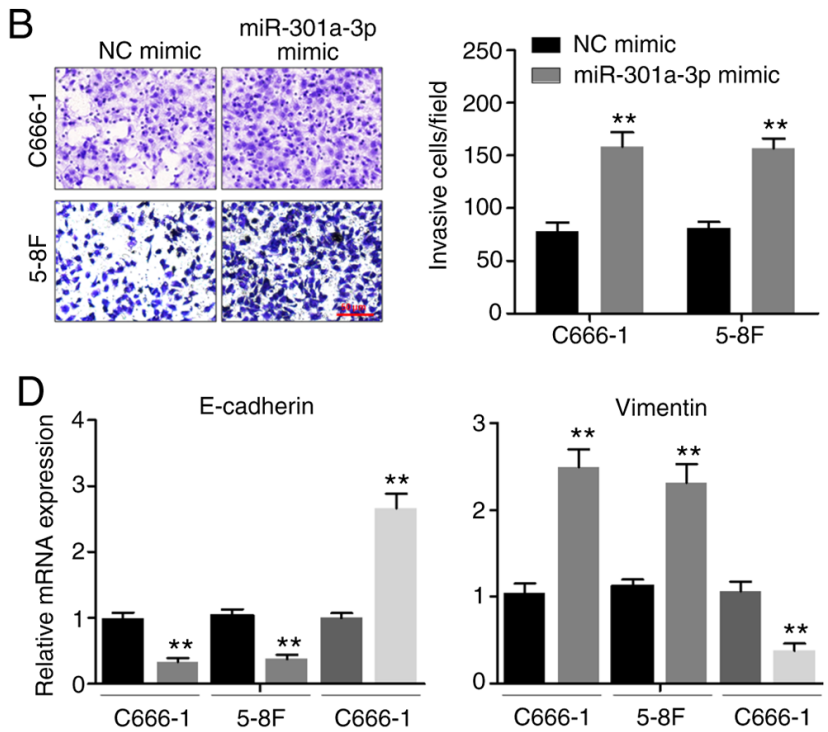
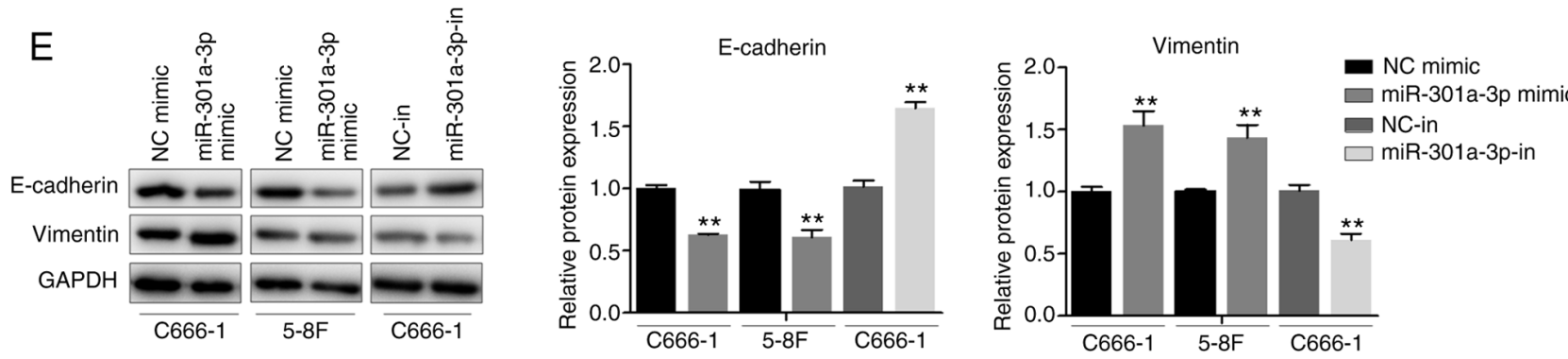

Figure 2. miR-301a-3p promotes cell migration, invasion and EMT of C666-1 and 5-8F cell lines. (A) Transwell migration assay of C666-1 and 5-8F cells transfected with miR-301a-3p mimic or NC. Magnification, x200. (B) Transwell invasion assay of C666-1 and 5-8F cells transfected with miR-301a-3p mimic or NC. Magnification, x200. (C) Transwell migration and invasion assays of C666-1 cells transfected with a miR-301a-3p inhibitor or NC. Magnification, x200. The (D) mRNA and (E) protein expression levels of EMT markers in the indicated cells as detected via reverse transcription-quantitative PCR and western blotting, respectively. " $\mathrm{P}<0.01$ vs. NC group. miR, microRNA; EMT, epithelial-mesenchymal transition; NC, negative control; in, inhibitor.

and colony-formation capacity (Fig. 1F). These data indicated that miR-301a-3p can enhance the proliferation of NPC cells.

miR-301a-3p promotes NPC cell migration, invasion and $E M T$ in vitro. To determine the effects of miR-301a-3p on the migration and invasion of NPC cells, the Transwell chamber assay was performed. miR-301a-3p overexpression significantly enhanced the invasive and migratory abilities of both C666-1 and 5-8F cells (Fig. 2A and B). By contrast, miR-301a-3p knockdown inhibited the invasive and migratory abilities of C666-1 cells (Fig. 2C). Since EMT is an important process during metastasis, the effects of miR-301a-3p on EMT was subsequently determined. The expression levels of both epithelial and mesenchymal markers were measured using RT-qPCR (Fig. 2D) and western blotting (Fig. 2E). It was observed that miR-301a-3p overexpression was associated with a significant downregulation of E-cadherin and a notable upregulation of vimentin, a mesenchymal marker. Furthermore, increased E-cadherin expression and decreased vimentin expression were observed following miR-301a-3p knockdown. Altogether, the observations demonstrated that miR-301a-3p plays a potential role in promoting NPC cell migration, invasion and EMT in vitro.

BTG1 is a putative direct target gene of miR-301a-3p. The potential regulatory targets of miR-301a-3p were explored via bioinformatics analysis of the miRanda and TargetScan databases. The analysis revealed $B T G 1$ as a likely target. One predicted binding site of miR-301a-3p was identified in the 3'-UTR region of BTG1 mRNA (Fig. 3A). RT-qPCR and western blotting assays revealed that miR-301a-3p overexpression downregulated the expression of BTG1 in C666-1 and 5-8F cells. In particular, miR-301a-3p overexpression decreased BTG1 mRNA and protein expression levels, whereas miR-301a-3p knockdown yielded an opposite effect (Fig. 3B and C). Moreover, luciferase reporter plasmids $B T G 1-3^{\prime} \mathrm{UTR}-\mathrm{Wt}$ and $B T G 1-3^{\prime} \mathrm{UTR}-\mathrm{Mt}$ were constructed, which were co-transfected in C666-1 and 5-8F cells with either miR-301a-3p mimic or NC. miR-301a-3p mimic suppressed the luciferase activity of the cells transfected with BTG1-3'-UTR-Wt, but not that of cells transfected with BTG1-3'-UTR-Mt (Fig. 3D). To further elucidate whether the effects of miR-301a-5p were mediated by repression of BTG1 in NPC cells, gain-of-function studies were performed. C666-1 cells were transfected with the BTGl plasmid without the 3'UTR region or vector control, and successful increase in BTG1 mRNA expression was verified by RT-qPCR (Fig. 3E). The BTG1 plasmid and miR-301a-3p mimic were then co-transfected into C666-1 cells, and RT-qPCR confirmed the expression of BTG1 mRNA (Fig. 3F). It was found that overexpression of $B T G 1$ inhibited miR-301a-3p mimic-induced cell proliferation and invasion in CCK-8, EdU and Transwell invasion assays (Fig. 3G-I). These observations demonstrated that BTG1 is potentially a direct target of miR-301a-3p. 
A

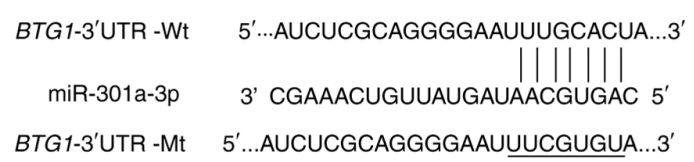

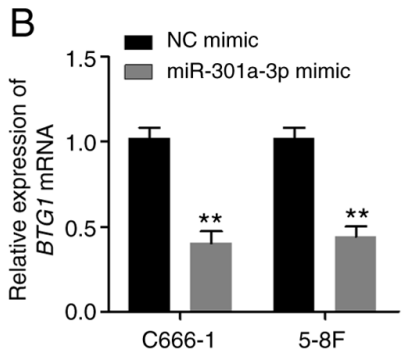
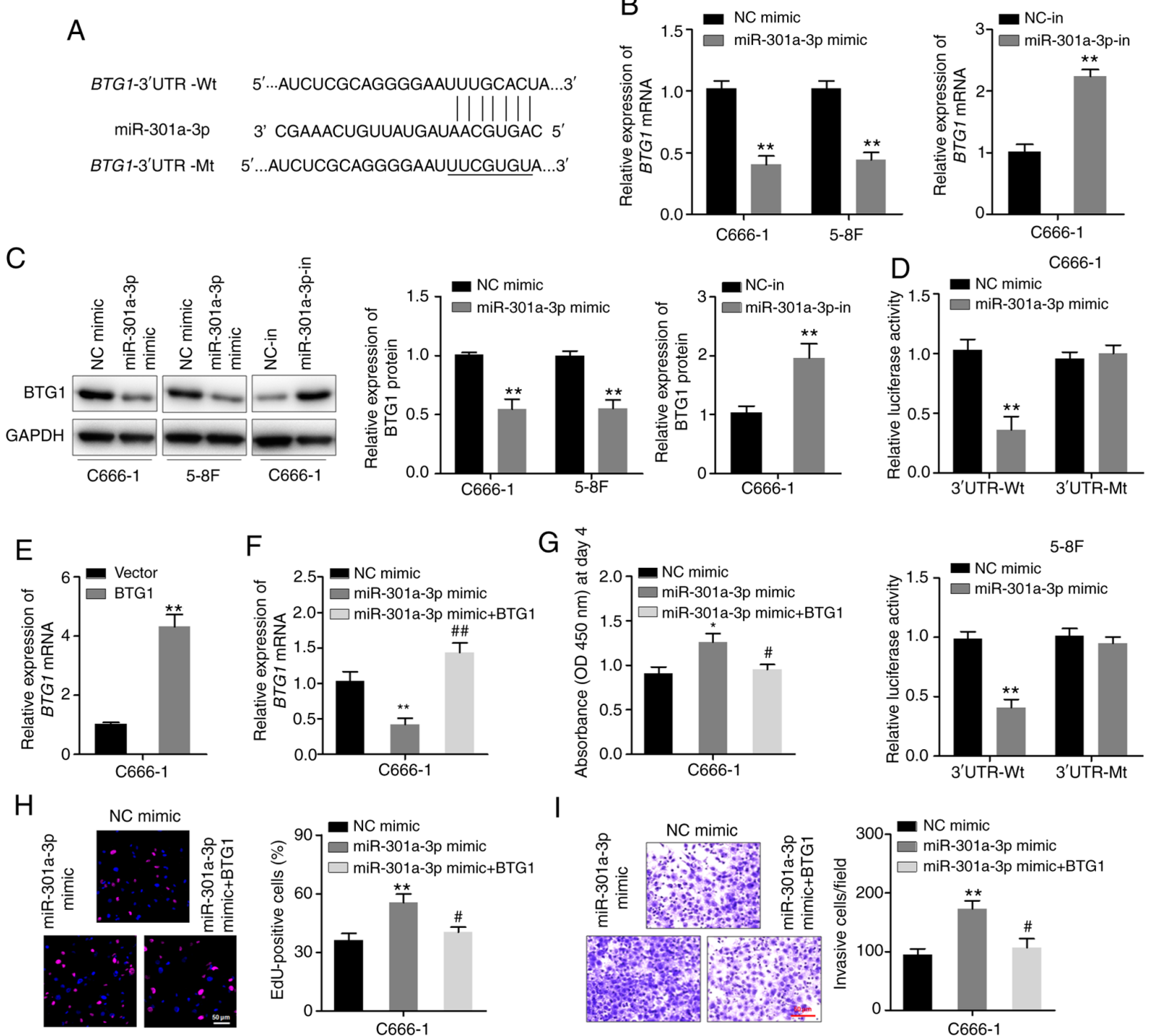

I
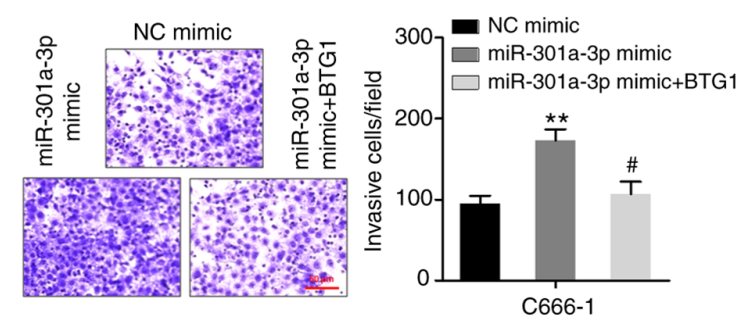

Figure 3. miR-301a-3p binds to the 3'-UTR of BTG1 mRNA. (A) The predicted miR-301a-3p-binding site and mutant miR-301a-3p-binding site in the 3'-UTR of BTG1 (present in the BTG1-3'-UTR-Wt and BTG1-3'-UTR-Mt plasmids, respectively). (B) RT-qPCR analysis of BTG1 expression of C666-1 and 5-8F cells transfected with the miR-301a-3p mimic and that of C666-1 cells transfected with the miR-301a-3p inhibitor. (C) Western blotting analysis of BTG1 expression in the indicated cells. (D) Luciferase activity of BTG1-3'-UTR-Wt or BTG1-3'-UTR-Mt plasmids in C666-1 and 5-8F cells after transfection with either the miR-301a-3p mimic or NC. (E) C666-1 cells were transfected with the BTG1 plasmid without the 3'-UTR region or vector control, and RT-qPCR was used to determine the expression of BTG1 mRNA. (F) C666-1 cells were co-transfected with the miR-301a-3p mimic and BTG1 expression plasmid. BTG1 mRNA levels were detected via RT-qPCR after 48 h. (G) Cell Counting Kit-8 assay. (H) EdU assay. (I) Transwell invasion assay. ${ }^{*} \mathrm{P}<0.05,{ }^{* * *} \mathrm{P}<0.01$ vs. vector or NC mimic group; ${ }^{\prime} \mathrm{P}<0.05$, ${ }^{\# /} \mathrm{P}<0.01$ vs. miR-301a-3p mimic group. miR, microRNA; UTR, untranslated region; BTG1, B-cell translocation gene-1; NC, negative control; in, inhibitor; RT-qPCR, reverse transcription-quantitative PCR; Wt, wild-type; Mt, mutant.

miR-301a-3p participates in cell-cell communication via exosomes. Finally, C666-1 and 5-8F cells were co-cultured with exosomes containing high miR-301a-3p levels, in order to determine whether this miRNA could be delivered via exosomes and regulate the function of recipient cells. Exosomes secreted from C666-1 cells stably overexpressing miR-301a-3p were isolated. TEM revealed that the exosomes had a lipid bilayer membrane structure, and western blotting confirmed the expression of exosomal markers CD63 and TSG101 (Fig. 4A and B). C666-1 and 5-8F cells were pretreated with the isolated exosomes, and RT-qPCR analysis confirmed that the expression levels of miR-301a-3p in NPC cells were significantly upregulated after treatment with exosomes derived from miR-301a-3p-overexpressing cells (miR-301a-exo) compared with control cells (NC-exo) (Fig. 4C). The CCK-8 and EdU assays revealed that miR-301a-exo significantly promoted the proliferation of C666-1 and 5-8F cells compared with NC-exo (Fig. 4D and E). Furthermore, the Transwell invasion assay indicated that miR-301a-exo significantly enhanced the invasion of NPC cells (Fig. 4F). These observations suggested that exosomal miR-301a-3p is absorbed by C666-1 and $5-8 \mathrm{~F}$ cells to participate in cell-cell communication to further regulate the malignant characteristics of NPC cells.

\section{Discussion}

The present study focused on the regulatory functions of miR-301a-3p in NPC cells. It was discovered that miR-301a-3p upregulation promotes the proliferation, migration, invasion and EMT of NPC cells in vitro. BTG1 mRNA was identified as 
A

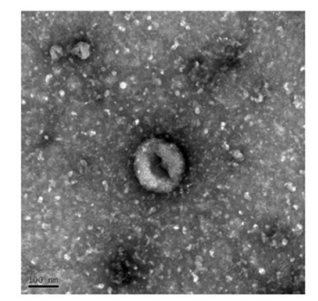

$\mathrm{E}$

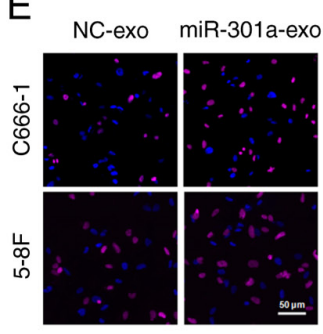

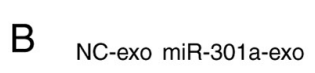

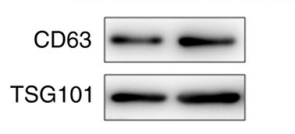

C

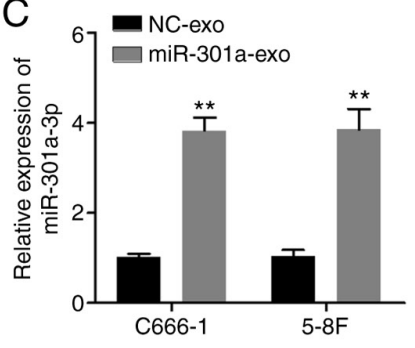

$\mathrm{F}$

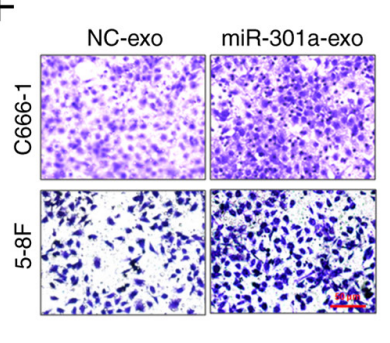

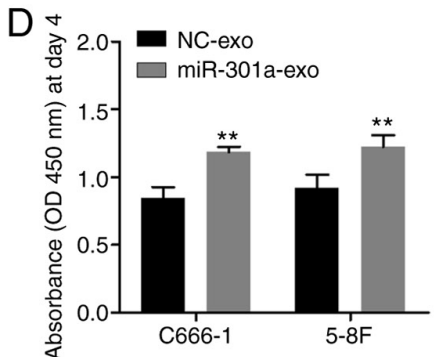

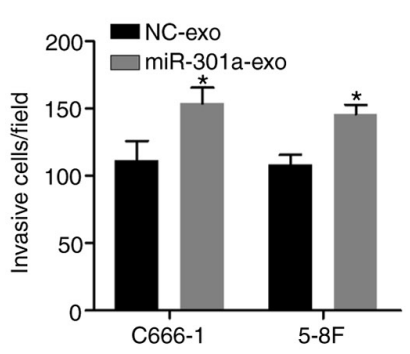

Figure 4. miR-301a-3p affects the proliferation, migration and invasion of nasopharyngeal carcinoma cell cells via exosomes. (A) Exosome identification via transmission electron microscopy. (B) Detection of the exosome marker proteins CD63 and TSG101 via western blotting. (C) Reverse transcription-quantitative PCR analysis of miR-301a-3p expression in C666-1 and 5-8F cells after treatment with miR-301a-exo or NC-exo. (D) Cell Counting Kit-8 assay to detect the viability of C666-1 and 5-8F cells after treatment with miR-301a-exo or NC-exo. (E) EdU assay to detect the proliferation of C666-1 and 5-8F cells after treatment with miR-301a-exo or NC-exo. Magnification, x200. (F) Transwell invasion assay. Magnification, x200. ${ }^{*} \mathrm{P}<0.05$; ${ }^{* *} \mathrm{P}<0.01$ vs. NC-exo group. miR, microRNA; BTG1, B-cell translocation; exo, exosome; NC, negative control.

the direct and functional target of miR-301a-3p in NPC cells. Furthermore, miR-301a-3p was delivered via exosomes, which promoted the proliferation and invasion of NPC cells. These findings indicate that the miR-301a-3p/BTG1 pathway plays important roles in the progression of NPC.

The aberrant expression of miR-301a-3p is associated with cancer initiation and development. miR-301a-3p has been reported to be upregulated in several types of cancer. For instance, Li et al (9) reported for the first time that miR-301a deficiency enhances $\mathrm{CD} 8^{+} \mathrm{T}$-cell accumulation in lung cancer cells by negatively regulating Runx3. Furthermore, Hu et al (10) showed that miR-301a promotes the invasion and metastasis of pancreatic cancer cells via the JAK/STAT3 signaling pathway by targeting SOCS5. Li et al (11) found that hyperglycemia-induced miR-301a promotes cell proliferation in prostate cancer cells by repressing p21 and Smad4. Nevertheless, the functions of miR-301a-3p in NPC cells remain unknown. In the present study, miR-301a-3p was overexpressed or knocked down in C666-1 and $5-8 \mathrm{~F}$ cells to evaluate the biological role of miR-301a-3p in NPC cells. It was confirmed that miR-301a-3p overexpression promotes the proliferation, migration, invasion and EMT of NPC cells in vitro, whereas miR-301a-3p knockdown inhibits the proliferation and invasion of these cells. These observations confirm that miR-301a-3p has an oncogenic function in NPC cells and support its potential use as a target of cancer therapy.

Existing experimental data suggest that miR-301a-3p directly regulates the expression of numerous target mRNAs, including Runx3, SOCS5, p21, Smad4, TCEAL7, FOXL1, ESR1 and PTEN (9-12,15-17). Therefore, it is essential to identify specific miR-301a-3p-mediated signaling pathways in NPC cells. TargetScan and miRanda database search identified the 3'-UTR region of BTG1 mRNA as the binding site of miR-301a-3p. Subsequently, BTG1 was identified as the critical target gene during experiments designed to unravel the biological role of miR-301a-3p in NPC. Furthermore, it was found that miR-301a-3p overexpression diminishes the mRNA and protein expression levels of BTG1 in vitro. The luciferase reporter assays in the present study also revealed that miR-301a-3p can directly bind to the 3'-UTR of BTG1 mRNA.

$B T G 1$, the key gene within the BTG/TOB family, is implicated in various biological phenomena, including cancer, and functions as a tumor suppressor gene (18). In a study on NPC, Sun et al (19) reported that BTG1 expression is downregulated in NPC cells and is possibly associated with tumor metastasis. Based on these findings, gain-of-function assays were conducted and it was determined that BTG1 overexpression partly reverses the tumor-promoting influence of miR-301a-3p. In other words, miR-301a-3p overexpression enhances the proliferation and invasion of human NPC cells by inhibiting a BTG1-mediated signaling pathway.

Finally, it was demonstrated that NPC cells transmit miR-301a-3p to surrounding cancer cells via exosomes and increased miR-301a-exo levels promote cell proliferation and invasion. Exosomes, secreted by various cell types, can be absorbed by the surrounding cells and exert their biological functions in these receptor cells (20). Exosomes with nucleic acids, including miRNAs, can contribute to cancer progression (21). A study demonstrated that hypoxic tumor-derived exosomal miR-301a promotes pancreatic cancer metastasis by mediating M2 macrophage polarization via the PTEN/PI3K $\gamma$ pathway (22). Exosomal miR-301a secreted from hypoxic glioma cells activates the Wnt/ $\beta$-catenin signaling pathway and promotes radiation resistance by targeting TCEAL7, and serum exosomal miR-301a concentration serves as a novel diagnostic biomarker of glioma and a prognostic factor of advanced-stage disease $(12,23)$. These findings suggest that exosomal miR-301a contributes to NPC tumorigenesis and progression. To the best of our knowledge, no study has evaluated the role of exosomal miR-301a-3p in NPC cells. Therefore, the present study analyzed miR-301a-3p levels in exosomes isolated from the cell 
culture supernatants of normal and NPC cells and observed high miR-301a-3p levels in NPC cell-derived exosomes. Next, it was determined whether miR-301a-3p exerts its functions via exosome delivery by treating C666-1 and 5-8F cells with the exosomes extracted from miR-301a-3p-overexpressing cells. Incubation with these highly expressed exosomes increased the proliferation and invasion of C666-1 and 5-8F cells. These results indicate that $\mathrm{miR}-301 \mathrm{a}-3 \mathrm{p}$ promotes the malignant parameters of NPC cells via exosome transfer.

In conclusion, the present data indicate that miR-301a-3p promotes the proliferation and invasion of human NPC cells by downregulating BTG1 and that this miRNA participates in cell-cell communication via exosomes. These findings provide a new direction for further investigating the pathogenesis of NPC. However, expression patterns of miR-301a-3p and survival outcomes of patients with NPC were not analyzed in the present study. Thus, these topics remain to be verified in future research.

\section{Acknowledgements}

Not applicable.

\section{Funding}

No funding was received.

\section{Availability of materials and data}

The datasets used and/or analyzed during the current study are available from the corresponding author on reasonable request.

\section{Authors' contributions}

QC and HJ made substantial contributions to the study conception and design. QC, QL and LX performed data acquisition, analyses and interpretation. QC drafted the manuscript and critically revised it for important intellectual content. QC and HJ confirm the authenticity of all the raw data. All authors read and approved the final manuscript.

\section{Ethics approval and consent to participate}

Not applicable.

\section{Patient consent for publication}

Not applicable.

\section{Competing interests}

The authors declare that they have no competing interests.

\section{References}

1. Chen W, Zheng R, Baade PD, Zhang S, Zeng H, Bray F, Jemal A, Yu XQ and He J: Cancer statistics in China, 2015. CA Cancer J Clin 66: 115-132, 2016.

2. Liang TS, Zheng YJ, Wang J, Zhao JY, Yang DK and Liu ZS MicroRNA-506 inhibits tumor growth and metastasis in nasopharyngeal carcinoma through the inactivation of the Wnt/ $\beta$-catenin signaling pathway by down-regulating LHX2. J Exp Clin Cancer Res 38: 97, 2019.
3. Kong YW, Ferland-McCollough D, Jackson TJ and Bushell M: microRNAs in cancer management. Lancet Oncol 13: e249-e258, 2012.

4. Filipowicz W, Bhattacharyya SN and Sonenberg N: Mechanisms of post-transcriptional regulation by microRNAs: Are the answers in sight? Nat Rev Genet 9: 102-114, 2008

5. Li B, Xu WW, Han L, Chan KT, Tsao SW, Lee NP, Law S, Xu LY, Li EM, Chan KW, et al: MicroRNA-377 suppresses initiation and progression of esophageal cancer by inhibiting CD133 and VEGF. Oncogene 36: 3986-4000, 2017.

6. Zheng Z, Qu JQ, Yi HM, Ye X, Huang W, Xiao T, Li JY, Wang YY, Feng J, Zhu JF, et al: MiR-125b regulates proliferation and apoptosis of nasopharyngeal carcinoma by targeting A20/NF- $\kappa B$ signaling pathway. Cell Death Dis 8: e2855, 2017.

7. Ye SB, Zhang H, Cai TT, Liu YN, Ni JJ, He J, Peng JY, Chen QY, Mo HY, Jun-Cui, et al: Exosomal miR-24-3p impedes T-cell function by targeting FGF11 and serves as a potential prognostic biomarker for nasopharyngeal carcinoma. J Pathol 240: 329-340, 2016.

8. Bao L, You B, Shi S, Shan Y, Zhang Q, Yue H, Zhang J, Zhang W, Shi Y, Liu Y, et al: Metastasis-associated miR-23a from nasopharyngeal carcinoma-derived exosomes mediates angiogenesis by repressing a novel target gene TSGA10. Oncogene 37: 2873-2889, 2018.

9. Li X, Zhong M, Wang J, Wang L, Lin Z, Cao Z, Huang Z, Zhang F, Li Y, Liu M, et al: miR-301a promotes lung tumorigenesis by suppressing Runx3. Mol Cancer 18: 99, 2019.

10. Hu H, Zhang Q, Chen W, Wu T, Liu S, Li X, Luo B, Zhang T, Yan G, Lu H, et al: MicroRNA-301a promotes pancreatic cancer invasion and metastasis through the JAK/STAT3 signaling pathway by targeting SOCS5. Carcinogenesis 41: 502-514, 2020.

11. Li X, Li J, Cai Y, Peng S, Wang J, Xiao Z, Wang Y, Tao Y, Li J, Leng Q, et al: Hyperglycaemia-induced miR-301a promotes cell proliferation by repressing p 21 and Smad4 in prostate cancer. Cancer Lett 418: 211-220, 2018

12. Yue X, Lan F and Xia T: Hypoxic glioma cell-secreted exosomal miR-301a activates $\mathrm{Wnt} / \beta$-catenin signaling and promotes radiation resistance by targeting TCEAL7. Mol Ther 27: 1939-1949, 2019.

13. Livak KJ and Schmittgen TD: Analysis of relative gene expression data using real-time quantitative PCR and the 2(-Delta Delta $\mathrm{C}(\mathrm{T})$ ) method. Methods 25: 402-408, 2001.

14. Lu J, Liu QH, Wang F, Tan JJ, Deng YQ, Peng XH, Liu X, Zhang B, Xu X and Li XP: Exosomal miR-9 inhibits angiogenesis by targeting MDK and regulating PDK/AKT pathway in nasopharyngeal carcinoma. J Exp Clin Cancer Res 37: 147, 2018.

15. Wang YG, Wang T, Shi M and Zhai B: Long noncoding RNA EPB41L4A-AS2 inhibits hepatocellular carcinoma development by sponging miR-301a-5p and targeting FOXL1. J Exp Clin Cancer Res 38: 153, 2019.

16. Lettlova S, Brynychova V, Blecha J, Vrana D, Vondrusova M, Soucek P and Truksa J: MiR-301a-3p suppresses estrogen signaling by directly inhibiting ESR 1 in ER $\alpha$ positive breast cancer. Cell Physiol Biochem 46: 2601-2615, 2018.

17. Xia X, Zhang K, Luo G, Cen G, Cao J, Huang K and Qiu Z: Downregulation of miR-301a-3p sensitizes pancreatic cancer cells to gemcitabine treatment via PTEN. Am J Transl Res 9: 1886-1895, 2017.

18. Yuniati L, Scheijen B, van der Meer LT and van Leeuwen FN: Tumor suppressors BTG1 and BTG2: Beyond growth control. J Cell Physiol 234: 5379-5389, 2019.

19. Sun GG, Wang YD, Cheng YJ and Hu WN: The expression of BTG1 is downregulated in nasopharyngeal carcinoma and possibly associated with tumour metastasis. Mol Biol Rep 41: 5979-5988, 2014

20. Kalluri R and LeBleu VS: The biology, function, and biomedical applications of exosomes. Science 367: eaau6977, 2020.

21. Kalluri R: The biology and function of exosomes in cancer. J Clin Invest 126: 1208-1215, 2016.

22. Wang X, Luo G, Zhang K, Cao J, Huang C, Jiang T, Liu B, Su L and Qiu Z: Hypoxic tumor-derived exosomal miR-301a mediates M2 macrophage polarization via PTEN/PI3K $\gamma$ to promote pancreatic cancer metastasis. Cancer Res 78: 4586-4598, 2018.

23. Lan F, Qing Q, Pan Q, Hu M, Yu H and Yue X: Serum exosomal miR-30la as a potential diagnostic and prognostic biomarker for human glioma. Cell Oncol (Dordr) 41: 25-33, 2018.

This work is licensed under a Creative Commons Attribution-NonCommercial-NoDerivatives 4.0 International (CC BY-NC-ND 4.0) License. 